\section{Análise do perfil das mães dos nascidos vivos em Carbonita, Minas, Gerais no ano de 1999, pelo estudo dos dados do "SINASC"}

\author{
Profile of mothers of the liveborn infants in \\ Carbonita, Minas Gerais, 1999 from \\ "SINASC" data
}

Walter Alvim de Albuquerque 1

Simone dos Santos Menezes 2

Haroldo da Silva Santana 3

1-3 Secretaria Municipal de Saúde de Carbonita, Minas Gerais Rua Belgo Mineira, 138, Monte Belo, Carbonita, Minas Gerais, Brasil, CEP.39.665-000, E mail: walteraa@uai.com.br

\begin{abstract}
Objectives: to compare the profile of mothers of liveborn infants in Carbonita in 1999 to the ones in Brazil and Minas Gerais. Through this analysis we intend to qualify the staff of Family Health Program, $(F H P)$ so they can be more effective.

Methods: the analysis was done based on mothers of babies born alive in Carbonita in the year1999. Registers of Community Health Agents, (RCHA) that have total knowledge about their area of action were also used. Data obtained from DATASUS was also used to compare Minas Gerais to Brazil.

Results: the analysis shows that most mothers in Carbonita have natural births and only 13,7\% are cesareans (in Brazil this attains 39,86\% and in Minas Gerais 46,43\%). It should be noted that children from Carbonita are not born with nourishment problems. Other important information refers to a high percentage of pregnant teenagers and women over 35. $\mathrm{Ob}$ serve that only $36 \%$ of pregnants went to 6 ideal minimum prenatal appointments. The mothers, who had completed Junior High School, are the ones who value prenatal the most.

Conclusions: the analysis shows that the staff of FHP must intensify the active search of pregnant women and improve the program of family planning to aim at reverting the health indicators.
\end{abstract}

Key words Natality, Pregnancy in adolescente, Delivery.

\section{Resumo}

Objetivo: comparar o perfil das mães das crianças nascidas vivas em Carbonita, Minas Gerais 1999, com as do Brasil e de Minas Gerais. Com esta análise pretende-se instrumentalizar as equipes do Programa de Saúde da Família (PSF), para atuarem de forma mais eficiente.

Métodos: a análise foi feita com dados das Declarações dos nascidos vivos (DN's), de mães residentes em Carbonita, no ano de 1999. Também utilizaram-se registros dos Agentes Comunitários de Saúde, (ACS) que têm total conhecimento do que ocorre em sua área de atuação. Utilizaram-se, ainda, dados obtidos do DATASUS, para efeito de comparação com Minas e com o Brasil.

Resultados: a análise mostra que a grande maioria dos partos das mães em Carbonita são normais e apenas 13,7\% são cesarianas, no Brasil atingem $39,86 \%$ e em Minas Gerais 46,43\%. Nota-se ainda que as crianças em Carbonita não nascem desnutridas. Outra importante informação se refere ao alto percentual de adolescentes grávidas e de gestantes acima de 35 anos de idade. Observa-se ainda, que apenas $36 \%$ das gestantes fizeram o mínimo ideal de 6 consultas de pré-natal. As mães com primeiro grau completo são as que mais valorizaram o pré-natal.

Conclusões: o estudo indica que as equipes do PSF devem intensificar a busca ativa de gestantes e melhorar o programa de planejamento familiar, visando reverter os indicadores apontados.

Palavras-chave Natalidade, Gravidez na adolescência, Parto. 


\section{Introdução}

Carbonita é um município situado no Vale do Jequitinhonha, no estado de Minas Gerais, a $421 \mathrm{Km}$ de Belo Horizonte e a $135 \mathrm{Km}$ de Diamantina. Tem $1.337 \mathrm{Km} 2$ de área geográfica e uma população estimada, para 2000, de 10.054 habitantes (Tabela 1).

Na Zona Rural predominam as residências dispersas, longe umas das outras, mas alguns pequenos aglomerados formam comunidades, como Santana, Abadia, Monte Belo e Mercadinho, onde é realizado um pequeno comércio de mercadorias e onde ficam instalados Postos de Saúde, Escola, Posto Telefônico, Igreja, entre outros poucos recursos.

Carbonita apresenta uma estrutura agrária de minifúndios, sendo que das 955 propriedades rurais, 915 têm até 100ha, como se pode observar na Tabela 2.

A população, até meados da década de 70 , era predominantemente rural (87\%), sobrevivendo com uma agropecuária rudimentar, apenas de subsistência. Atualmente a população rural não ultrapassa os $40 \%$ (Tabela 1). Na década de 70 instalou-se em Carbonita a empresa Companhia AgroFlorestal Santa Bárbara (CAF Santa Bárbara), que veio revolucionar a economia do município e deixar marcas indeléveis na sua história. Essa empresa, na época um departamento da Aços Especiais Itabira (ACESITA), contratou muitos funcionários para promover o plantio de eucalipto para produção do carvão vegetal.

Em 1976 tinha aproximadamente 1.200 funcionários diretos, fato que promoveu uma urbanização crescente bem como atração sobre a mão-deobra dos municípios vizinhos. Hoje, a CAF, praticamente desativada, terceirizou seu trabalho para empreiteiras que absorveram parte da mão-de-obra demitida.

Paralelamente a este contexto aconteceu a municipalização da saúde no Brasil, que transferiu às autoridades municipais e à comunidade local a responsabilidade de definir os rumos e as prioridades dos programas de saúde em cada município.

A partir de 1993 o Ministério da Saúde propôs a estratégia de Programa de Saúde da Família (PSF), como forma de realizar a atenção primária à saúde, estimulando a implantação das equipes - responsabilidade do município - mas recebendo apoio técnico e financeiro de Secretarias Estaduais de Saúde e Ministério da Saúde.

O Programa de Saúde da Família é constituído por equipes que têm em sua composição básica um médico, uma enfermeira, uma auxiliar de enfermagem e seis agentes comunitários de saúde (ACS). As equipes são implantadas em áreas geográficas
Tabela 1

População de Carbonita no período 1970-2000

\begin{tabular}{rccc}
\hline Ano & Urbano & Rural & Total \\
\hline 1970 & 927 & 6.032 & 6.959 \\
1980 & 2.369 & 5.902 & 8.271 \\
1991 & 3.631 & 4.564 & 8.195 \\
1996 & 5.593 & 3.492 & 9.085 \\
2000 & - & - & 10.054 \\
\hline
\end{tabular}

Fonte: Instituto Brasileiro de Geografia e Estatística, IBGE

Tabela 2

Distribuição das propriedades rurais conforme área (ha)

\begin{tabular}{lc}
\hline Área (ha) & Quantidade \\
\hline 1 a 10 & 444 \\
11 a 100 & 471 \\
101 a 500 & 35 \\
501 a 1.000 & 2 \\
+ de 1.000 & 3 \\
Total & 95 \\
\hline
\end{tabular}

Fonte: Escritório local da EMATER, Minas Gerais

bem delimitadas e são responsáveis pela saúde de cerca de 600 a 1.000 famílias, ou de 2.400 a 4.000 habitantes.

Nos anos mais recentes, tem havido aumento de interesse pelo tema da avaliação em saúde observado tanto no âmbito dos serviços, quanto dos próprios usuários e familiares. ${ }^{1}$ Esse interesse foi incrementado nos últimos dois anos no âmbito dos serviços a partir do momento em que o Ministério da Saúde propôs um Pacto de Metas, ${ }^{2}$ a ser trabalhado por todos os municípios que prestam atenção básica, ou tenham implantado o Programa de Saúde da Família e/ou o Programa de Agentes Comunitários de Saúde (PACS).

Para a avaliação do Pacto de Metas são usados indicadores extraídos dos vários sistemas de informação de base nacional existentes. ${ }^{3} \mathrm{Um}$ desses sistemas é o Sistema de Informação de Nascidos Vivos (SINASC).

O SINASC foi implantado no Brasil em 1990,4 com características semelhantes de outros países co- 
mo EUA, Cuba, Suécia e Itália, e se baseia no preenchimento das Declarações de Nascidos Vivos (DN's), no serviço de saúde onde ocorre o parto, ou no cartório de Registro Civil, quando a criança nasce em parto domiciliar. Em Carbonita, tal sistema só foi efetivamente implantado a partir de 1998, quando se iniciou o processo de sistematização das ações de saúde e valorização dos dados estatísticos para fins de programação, controle e avaliação dos trabalhos executados. Usou-se para nascido vivo o conceito definido pela Organização Mundial da Saúde (OMS), citado em Mello Jorge (1992: 18) 5 de " ... todo produto da concepção que, independentemente do tempo da gestação, depois de expulso ou extraído do corpo da mãe, respire ou apresente outro sinal de vida, tal como batimento cardíaco, pulsação do cordão umbilical ou movimentos efetivos dos músculos de contração voluntária, estando ou não desprendida a placenta".

\section{Métodos}

Esta análise foi feita com os dados oriundos das DN's de mães residentes em Carbonita, do ano de 1999. Sabe-se, todavia, que os sistemas de informação têm vários problemas: na coleta de dados, no processamento e análise, e principalmente na subnotificação. ${ }^{3}$ Nesse sentido, além das DN's originadas no Serviço de Saúde local, foram pesquisadas ainda aquelas preenchidas no Cartório de Registro Civil e nos municípios vizinhos que são referência para o serviço de obstetrícia. Das DN's originadas no município excluiram-se aquelas em que as mães não residem em Carbonita. Nesse município, o PSF cobre $100 \%$ de sua área e os ACS têm total controle das crianças nascidas em sua área de atuação. Foi feita busca ativa dos nascidos vivos comparando com as DN's existentes e corrigindo-as quando necessário; portanto, o número pode ser considerado bastante fiel à realidade. A diferença entre a informação dos ACS e das DN's computadas é de $2,7 \%$ Foram analisadas 175 DN's fazendo cruzamento dos dados disponíveis.

Para esse estudo, além de uma revisão bibliográfica, foram utilizadas informações obtidas da Internet, ${ }^{6}$ para efeito de comparação com o estado de Minas Gerais e com o Brasil, apesar de os dados disponíveis serem do ano de 1997. Ocasionalmente, comparou-se com o Norte de Minas e Montes Claros, regiões com perfil sócioeconômico semelhantes ao de Carbonita.

\section{Resultados}

Pelos dados da Figura 1 podemos notar que as crianças de Carbonita não nascem desnutridas, se comparadas com os dados de Minas Gerais e do Brasil.

Podemos verificar que, na grande maioria os partos das mães de Carbonita são normais $(85,70 \%)$; apenas $13,70 \%$ são partos cesáreos, enquanto que no Brasil e em Minas Gerais o número de partos cesáreos é bem maior $(39,86 \%$ no Brasil e $46,43 \%$ em Minas Gerais) (Tabela 3).

Segundo a faixa etária das mães dos nascidos vivos (Figura 2) podemos notar que, em comparação com o Brasil e Minas Gerais, Carbonita apresenta um percentual maior que o índice estadual e o federal na faixa das adolescentes, mas o que salta aos olhos é na faixa acima de 35 anos, onde os índices desse município chegam ao dobro do índice do Estado e do Brasil.

$\mathrm{Na}$ análise do percentual de consultas de pré-natal realizadas pelas mães de Carbonita (Tabela 4) podemos notar que apenas $2,86 \%$ a realizaram.

Quanto ao número absoluto de consultas (Figura 3 ) podemos notar uma tendência levemente ascendente, para mais de 6 consultas e descendente para até 6 consultas.

$\mathrm{Na}$ Tabela 5 podemos notar que as mães com $1^{\circ}$ grau completo são as que mais valorizam o pré-natal, comparecendo a mais de 6 consultas durante a gestação, enquanto nos outros graus de estudo a frequiência aos pré-natais foi bem menor.

\section{Figura 1}

Nascidos vivos por peso em Carbonita, Minas Gerais e Brasil

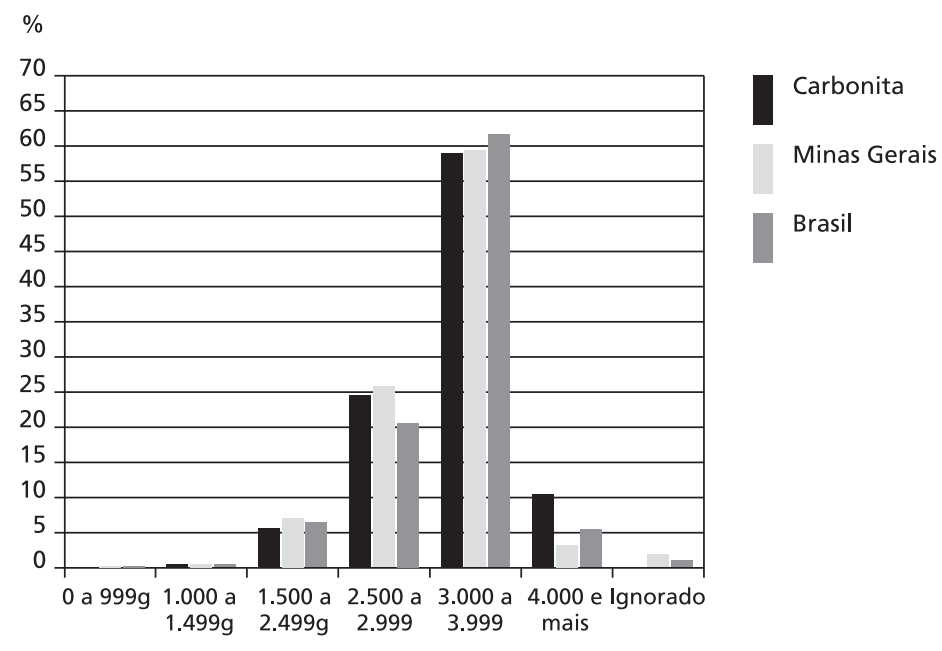

Fonte: Ministério da Saúde e Secretaria Municipal de Saúde de Carbonita, Minas Gerais 
Tabela 3

Percentual dos nascidos vivos por tipo de parto no Brasil, Minas Gerais e Carbonita

\begin{tabular}{lccc}
\hline & $\begin{array}{c}\text { Brasil } \\
\%\end{array}$ & $\begin{array}{c}\text { Minas Gerais } \\
\%\end{array}$ & $\begin{array}{c}\text { Carbonita } \\
\%\end{array}$ \\
\hline Normal & 58,19 & 51,23 & 85,70 \\
Cesáreo & 39,86 & 46,43 & 13,70 \\
Fórceps & 0,98 & 1,15 & 0,60 \\
Outros & 0,04 & 0,08 & 0,00 \\
Ignorado & 0,93 & 1,11 & 0,00 \\
\hline
\end{tabular}

Fonte: Ministério da Saúde e Secretaria Municipal de Saúde de Carbonita, Minas Gerais

Tabela 4

Percentual no número de consultas de pré-natal por faixa etária

\begin{tabular}{lccccc}
\hline \multirow{2}{*}{$\begin{array}{c}\text { Idade } \\
\text { da Mãe }\end{array}$} & \begin{tabular}{c} 
Nenhuma \\
\cline { 2 - 6 }
\end{tabular} & Até & Mais de & Ignorado & Total \\
\cline { 2 - 6 } & $\%$ & $6 \%$ & $6 \%$ & $\%$ & $\%$ \\
\hline a 14 anos & 0,00 & 1,14 & 0,57 & 0,00 & 1,71 \\
15 a 19 anos & 0,00 & 12,00 & 6,29 & 0,00 & 18,29 \\
20 a 34 anos & 2,29 & 38,29 & 21,71 & 0,00 & 62,29 \\
35 anos e mais & 0,00 & 9,14 & 6,86 & 0,00 & 16,00 \\
Ignorado & 0,57 & 0,57 & 0,57 & 0,00 & 1,74 \\
Total & 2,86 & 61,14 & 36,00 & 0,00 & 100,00 \\
\hline
\end{tabular}

Fonte: Secretaria Municipal de Saúde de Carbonita, Minas Gerais

\section{Tabela 5}

Número e proporção dos nascidos vivos por grau de instrução da mãe, segundo quantidade de consultas pré-natal

\begin{tabular}{|c|c|c|c|c|c|c|c|c|}
\hline \multirow{3}{*}{$\begin{array}{c}\text { Grau de Instrução } \\
\text { da mãe }\end{array}$} & \multicolumn{8}{|c|}{ Quantidade de consultas de pré-natal } \\
\hline & \multicolumn{2}{|c|}{ Nenhum } & \multicolumn{2}{|c|}{ Até 6} & \multicolumn{2}{|c|}{ Mais de 6} & \multicolumn{2}{|c|}{ Total } \\
\hline & $\mathbf{n}$ & $\%$ & $\mathbf{n}$ & $\%$ & $\mathbf{n}$ & $\%$ & $\mathbf{n}$ & $\%$ \\
\hline Nenhum & 2 & 12,50 & 8 & 50,00 & 6 & 37,50 & 16 & 100,00 \\
\hline $1^{\circ} \mathrm{Grau}$ Incompleto & 2 & 1,65 & 80 & 66,12 & 39 & 32,23 & 121 & 100,00 \\
\hline $1^{\circ} \mathrm{Grau}$ Completo & 0 & 0,00 & 7 & 38,89 & 11 & 61,11 & 18 & 100,00 \\
\hline $2^{\circ} \mathrm{Grau}$ & 1 & 6,25 & 9 & 56,25 & 6 & 37,50 & 16 & 100,00 \\
\hline Superior & 0 & 0,00 & 0 & 0,00 & 0 & 0,00 & 0 & 0,00 \\
\hline Ignorado & 0 & 0,00 & 3 & 75,00 & 1 & 25,00 & 4 & 100,00 \\
\hline Total & 5 & 2,86 & 107 & 61,14 & 63 & 36,00 & 175 & 100,00 \\
\hline
\end{tabular}

Fonte: Secretaria Municipal de Saúde de Carbonita, Minas Gerais 
Nascidos vivos por peso em Carbonita, Minas Gerais e Brasil

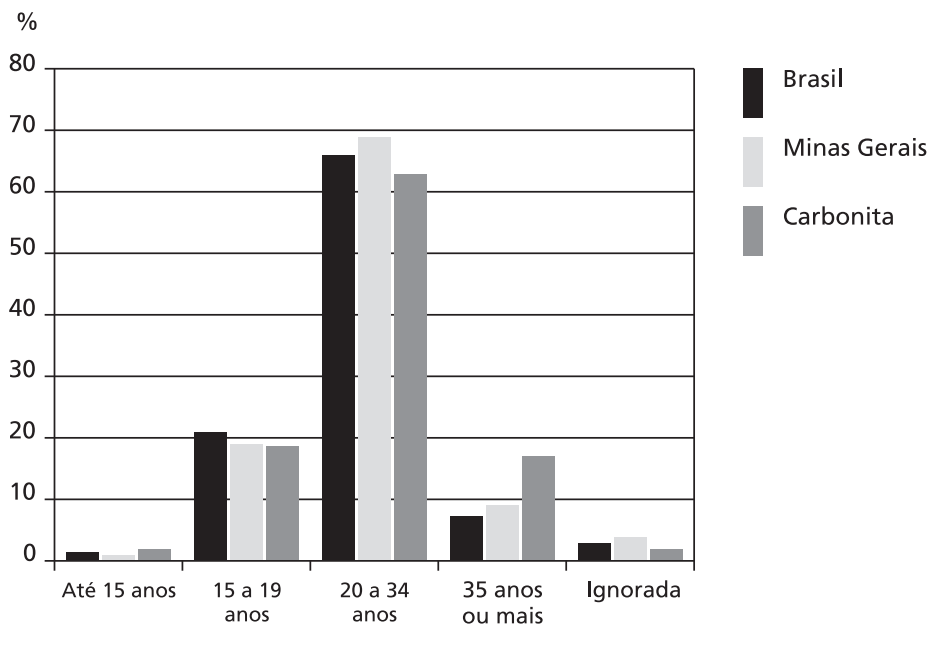

Fonte: Ministério da Saúde e Secretaria Municipal de Saúde de Carbonita, Minas Gerais

Figura 3

Número absoluto de consultas de pré-natal por faixa etária

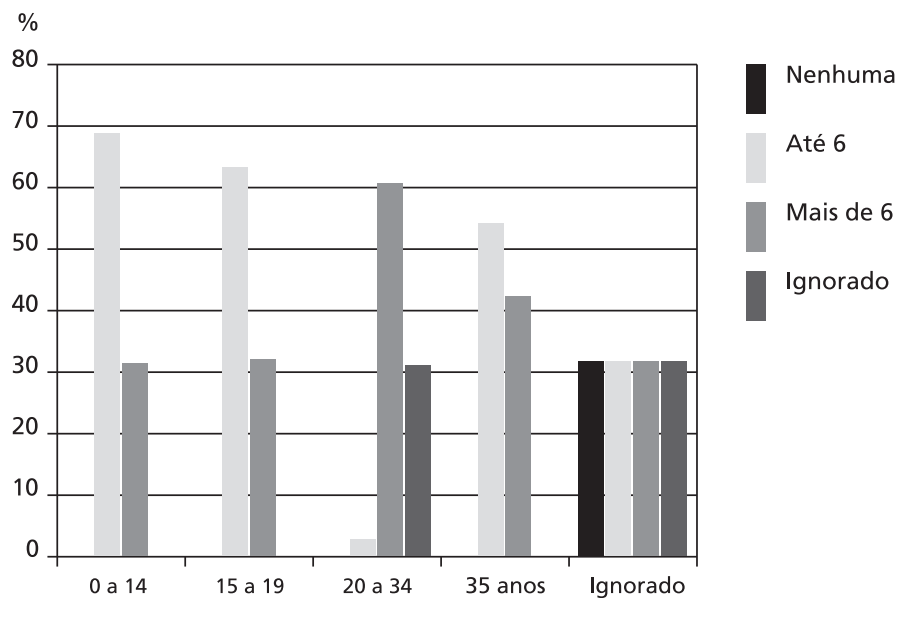

Fonte: Secretaria Municipal de Saúde de Carbonita, Minas Gerais 


\section{Discussão}

A análise dos dados do SINASC de Carbonita no ano de 1999, mostra uma situação inquietante para os profissionais que atuam no município. Primeiro, o percentual de adolescentes grávidas é na faixa etária acima de 35 anos, quando engravidar volta a ser uma situação de risco, em Carbonita as mães engravidam duas vezes mais que no Brasil e no Estado. Os dados nos mostram ainda que o número de consultas de pré-natal realizadas foi bem menor do que o mínimo de 7 consultas, o que uma necessidade de sensibilizar melhor a gestante para a importância do pré-natal. Por outro lado podemos notar um índice altíssimo de partos normais $(85,70 \%)$, número significativamente maior que os do Brasil e os de Minas Gerais. Outro dado importante se refere ao peso dos recém-nascidos, que não difere, praticamente, do peso dos recém-nascidos do Brasil e de Minas Gerais, podendo-se concluir que a desnutrição infantil em Carbonita acontece após o nascimento por outras causas, o que merece estudo posterior.

Em Montes Claros e no Norte de Minas os partos naturais corresponderam a $70 \%$ e $79,8 \%$, e as cesáreas a $30 \%$ e $20 \%$ respectivamente. 7 O relatório "A saúde no Brasil" da Organização Pan-Americana de Saúde (OPAS; 2000: 13) $)^{8}$ mostra que " ... a percentagem de partos cesáreos permanece significativamente elevada, tendo aumentado de 32\% em 1986 para 36\% em 1996". Ao que tudo indica, parece haver na referência obstétrica de Carbonita a utilização da cesárea apenas nos casos tecnicamente indicados ou nas urgências.

Estudando os nascidos vivos na cidade de Passos, Minas Gerais, - situada em região sócioeconômica muito melhor que Carbonita, Maia, ${ }^{9}$ encontrou uma taxa de baixo peso de $10 \%$. A desnutrição que já foi constatada no município se deve, provavelmente, à baixa taxa de amamentação exclusiva e ao desmame precoce, aliada às baixas condições sócioeconômicas da população. Talvez o que explique essa similitude de baixo peso ao nascer, mesmo nas condições tão adversas encontradas no Jequitinhonha, seja o trabalho desenvolvido na assistência ao pré-natal pelas equipes de saúde da família.

$\mathrm{O}$ fato de Carbonita apresentar um alto índice de partos na faixa etária acima de 35 anos, certamente reflete o pequeno acesso que a mulher tem aos meios anticoncepcionais mais adequados para esta faixa etária, como a laqueadura e o dispositivo intra-uterino (DIU), além da disponibilidade irregular dos anovulatórios no serviço de saúde. Correia e McAuliffe (1994: 329), 10 afirmam que o "... perfil de saúde da mulher no Brasil está longe de ser considerado satisfatório." Apesar dos avanços obtidos com a implantação do PSF e depois com o incipiente Programa de Saúde da Mulher, o município ainda parece estar longe de solucionar esses problemas.

Os dados mostram que as mães de Carbonita tiveram um alto índice de cobertura de pré-natal. Em estudo realizado na cidade do Rio de Janeiro, foi encontrado $67,8 \%$ e $76,2 \%$ de cobertura para os anos de 1995 e 1996.11 Todavia, apenas 36\% delas atingiram o mínimo ideal de mais de 6 consultas. No site do Ministério da Saúde, 6 essa informação não está disponível, o que nos impede de comparar Carbonita com Minas e com o Brasil. No estudo referente ao Norte de Minas, encontrou-se 18,3\% das gestantes que não fizeram sequer uma consulta no pré-natal enquanto apenas $38,9 \%$ tiveram entre 5 e mais consultas. Podemos observar, ademais, que quanto maior a faixa etária da gestante, mais importância ela dá à consulta de pré-natal. 


\section{Referências}

1. Carvalho AL, Amarante P. Avaliação de qualidade dos novos serviços de saúde mental - em busca de novos parâmetros. Saúde Debate 1996; 52: 74-82.

2. Ministério da Saúde. Pacto de metas. Portaria 476/GM de 14 de abril de 1999. Diário Oficial da União (DOU), nº 122 de 28 de junho de 1999.

3. Carvalho DM. Grandes sistemas nacionais de informação em saúde: revisão e discussão da situação atual. Inf Epidemiol SUS 1997; 4: 7-46.

4. Mello Jorge HP, Gotlieb SLD, Oliveira H. O sistema de informações sobre nascidos vivos: primeira avaliação dos dados brasileiros. Inf Epidemiol SUS 1996; 5: 15-48.

5. Mello Jorge HP et al. O sistema de informações sobre nascidos vivos - SINASC. São Paulo: NEPS Universidade de São Paulo; 1992. [Série Divulgação, 7] apud Carvalho DM. Grandes sistemas nacionais de informação em saúde: revisão e discussão da situação atual. Inf Epidemiol SUS 1997; 4: 18

6. Ministério da Saúde. Dados e indicadores de saúde. Disponível em: http://www.datasus.gov.br/cgi/tabcgi.exesinasc/dxmg.def [jun 2000].
7. Minas Gerais. Secretaria de Saúde. Projeto de redução da mortalidade na infância. Belo Horizonte: Secretaria Estadual de Saúde; 1993. [mimeo]

8. OPAS. Organização Pan-Americana da Saúde. A saúde no Brasil. Brasília, DF: OPAS; 1998. 48p

9. Maia MA. Characterization of liveborn infants at hospitals in the first year of implementation of the information subsystem on liveborn infants in a municipality of Minas Gerais, Brazil; 1997. Disponível em: http://search.healthgate.com/cgi-bin/q-format.cgi [nov 2000]

10. Correia LL, McAuliffe JF. Saúde materno-infantil. In: Rouquayrol MZ, organizador. Epidemiologia \& saúde. São Paulo: MEDSI; 1994. p. 315-42.

11. Silva RI, Theme FMM, Noronha CP. Sistema de informação sobre nascidos vivos na cidade do Rio de Janeiro: 1993/1996. Inf Epidemiol SUS 1997; 2: 33-48. 